6 American Thoracic Society, CDC, Infectious Diseases Society of America. Treatment of tuberculosis. MMWR Recomm Rep 2003; 52: 1-77.

7 de Kantor IN, Ambroggi M, Poggi S, et al. Human Mycobacterium bovis infection in ten Latin American countries. Tuberculosis 2008; 88: 358-365.

8 World Health Organization. Treatment of Tuberculosis Guidelines. 4th Edn. Geneva, World Health Organization 2010 .

9 Cicero R, Olivera H, Hernández-Solis A, et al. Frequency of Mycobacterium bovis as an etiologic agent in extrapulmonary tuberculosis in HIV-positive and -negative Mexican patients. Eur J Clin Microbiol Infect Dis 2009; 28: 455-460.

10 Kataria YP. Observations on human infection with Mycobacterium bovis. Tubercle 1969; 50: 14-21.

11 O'Donohue WJ Jr, Bedi S, Bittner MJ, et al. Short-course chemotherapy for pulmonary infection due to Mycobacterium bovis. Arch Intern Med 1985; 145: 703-705.

12 Esteban J, Robles P, Soledad Jiménez M, et al. Pleuropulmonary infections caused by Mycobacterium bovis: a re-emerging disease. Clin Microbiol Infect 2005; 11: 840-843.

13 Sauret J, Jolis R, Ausina V, et al. Human tuberculosis due to Mycobacterium bovis: report of 10 cases. Tuber Lung Dis 1992; 73: 388-391.

14 LoBue PA, Moser KS. Treatment of Mycobacterium bovis infected tuberculosis patients: San Diego County, California, United States, 1994-2003. Int J Tuberc Lung Dis 2005; 9: 333-338.

15 Cordova E, Gonzalo X, Boschi A, et al. Human Mycobacterium bovis infection in Buenos Aires: epidemiology, microbiology and clinical presentation. Int J Tuberc Lung Dis 2012; 16: 415-417.

16 Majoor CJ, Magis-Escurra C, van Ingen J, et al. Epidemiology of Mycobacterium bovis disease in humans, the Netherlands, 1993-2007. Emerg Infect Dis 2011; 17: 457-463.

\title{
Faster for less: the new "shorter" regimen for multidrug-resistant tuberculosis
}

To the Editor:

Multidrug resistant (MDR) and extensively drug-resistant (XDR) tuberculosis (TB) are growing clinical and public health concerns, with an estimated worldwide incidence and mortality of 480000 and 190000 cases, respectively (2014) [1]. The World Health Organization (WHO) End TB Strategy reiterates the MDR-/XDR-TB threat and the solutions to control the epidemic [2]. Unfortunately, large proportions of patients with resistant $\mathrm{TB}$ do not have access to adequate diagnostics and treatment yet, while treatment success rates remain suboptimal (as demonstrated in the largest retrospective cohort of MDR-TB patients, i.e., TB caused by Mycobacterium tuberculosis isolates resistant to at least isoniazid and rifampicin) and decrease further with resistance patterns beyond XDR-TB [3].

Presently, several of the available drugs have limited efficacy, being either toxic or unobtainable or both, and the treatment may take up to 24 months or longer. Although a few, new and repurposed drugs are fortunately available, clinicians often have difficulties in designing effective regimens [4], due to lack of drugs and rapid diagnostics, susceptibility results, comorbidities, drug toxicities and tolerability.

Recently, WHO published new recommendations aimed at speeding up TB second-line drug resistance detection (rapid molecular MTBDRsl test) and improving treatment outcomes of MDR-TB cases (shorter MDR-TB regimen) [5]. This is a demonstration of the efforts urgently being made to provide wider access to diagnosis and treatment in countries with the highest burden of MDR-TB. WHO has highlighted the advantages of the new regimen (consisting of 4-6 months of kanamycin, moxifloxacin, prothionamide, clofazimine, pyrazinamide, high-dose isoniazid and ethambutol followed by 5 months of moxifloxacin, clofazimine, pyrazinamide and ethambutol), providing a fact sheet with the necessary explanations. They include its shorter duration (9-11 months), which will improve adherence and its "relatively" low cost $(<1000$ US dollars per patient), which will ensure sustainability; these features are extremely important in resource-limited settings and in rich countries. It is possible that the antibiotic regimen may be modified: for example kanamycin is likely to be replaced by capreomycin or amikacin and these modifications may increase the overall cost of the regimen [6].

The regimen is recommended for MDR-TB cases not resistant to, and never treated with second-line anti-TB drugs and, therefore, should not be used if there is a documented or suspected resistance or previous use of one of the drugs composing the regimen [5]. The new push towards broader molecular testing at an earlier stage will enable patients to be selected for the shorter MDR-TB regimen more readily 
and safely by reducing the window of resistance, laboratory errors and turn-around times. If resistances are present for one or more drugs in the shorter regimen then it could be argued that, possibly, these could be replaced with linezolid, delamanid or bedaquiline and still maintain a shorter treatment duration; however, there is not sufficient evidence presently available to recommend this [5].

Resistance to pyrazinamide, even if determined by reliable drug-susceptibility testing (DST) is not an absolute contraindication for the shorter MDR-TB regimen, unless there are accompanying elements indicating that one or more other agents in the regimen are also ineffective. It is not recommended to base treatment decisions on the DST for ethambutol owing to the unreliable nature of the test. There is no reliable DST available for clofazimine or prothionamide at this stage.

A question clinicians will ask is whether the shorter MDR-TB regimen is likely to work in all settings and especially outside trial conditions. However, the fundamentals of the shorter regimens are practically the same of the previous conventional 24-month regimens. They are using practically the same number of drugs, including a fluoroquinolone, a second-line injectable, and two other companion drugs. The only difference is that the fluoroquinolone should be moxifloxacin (used in many conventional 24-month MDR-TB regimens), and the replacement of cycloserine with clofazimine.

The International Carbapenems Study Group recently carried out a multi-centre, observational, retrospective, cohort study performed in centres specialised in the management of MDR-/XDR-TB cases in 11 countries, out of which eight (72.7\%) were in Europe and three (27.3\%) were in South America, including 348 patients in total [7-9]. Individuals aged $<15$ years were excluded. Only adults with a culture-confirmed diagnosis of MDR-TB were enrolled, and evaluated according to meropenem/ imipenem-containing and -sparing regimens. The prevalence of resistances to the drugs included in the "shorter MDR-TB regimen" is summarised in table 1. We noted high proportional resistance to ethambutol and pyrazinamide $(>60 \%$,) prothionamide $(55.4 \%$;), fluoroquinolones $(40.8 \%)$ and kanamycin (44.4\%) (there were no data on clofazimine or high-dose isoniazid). In South America, we noticed higher prevalence of resistance to fluoroquinolones (86.8\%) and kanamycin (67.9\%), probably due to the selection of complicated cases in reference centres, with higher proportions of retreatment cases compared with the European patients.

Our results suggest that a shorter MDR-TB regimen in our cohort would have an impact on only a minority of patients and may have limited use in these settings where patients have more resistant forms of $\mathrm{TB}$ and are more treatment experienced (like in reference centres); only 14 (4.0\%) out of 348 new and retreated patients were susceptible to all the shorter MDR-TB regimen drugs (high-dose isoniazid and clofazimine resistance is unknown as both not routinely tested).

The lack of susceptibility to the new regimen was replicated even in the new patients' subgroup in our cohort (145 patients). Our study has a number of limitations given that it is retrospective, our cohort is super selected with a large representation of resistant cases with few therapeutic options, and is by definition not representative of the European and Latin America settings.

The study supporting the WHO recommendation is solid, being based on a multi-centre study including 1200 patients. The WHO analysis provides a strong evidence-based backbone to the implementation of this innovative regimen. It will favour an improvement of patient adherence and of drug safety and tolerability profile. However, a cautious decision-making approach, based on DST, is necessary, particularly in "hot spots" for MDR-/XDR-TB (e.g., former Soviet Union Countries) in new and previously treated cases. The "shorter MDR-TB regimen" will be a useful tool in the fight against MDR-/XDR-TB if properly utilised.

Critics are concerned that the programmatic management of MDR-TB with a shorter MDR-TB regimen may in turn lead to the selection of XDR-TB cases; however, at present, there is no evidence to substantiate for this, as shorter regimens have produced excellent outcomes under operational research conditions in some settings [10-12]. The opposite may be true, as the availability of a shorter MDR-TB regimen will allow for more patients to be treated and increase the chances of completing treatment, and this ultimately will reduce numbers of MDR-TB patients and the prevalence of XDR-TB patients over time. Currently the strongest risk factor for a bacteriologically unfavourable outcome with the shorter MDR-TB regimen consists of high-level fluoroquinolone resistance, particularly when compounded by initial pyrazinamide resistance [12]. If local epidemiology is known and rapid MTBDRsl testing used to ensure susceptibility for the key drugs composing the regimen, the shorter MDR-TB regimen could be very important for some patients as treatment duration is significantly reduced. To further safeguard the regimen, drug exposure may be evaluated. This will reduce the chance of development of drug resistance. Nowadays, simple tools are available which limit the use of therapeutic drug monitoring not only to the reference centre [13]. Apart from the selection of treatment based on DST it will then be up to the clinician to tailor the regimen based on the extensiveness of the disease, its location, monitoring of 
TABLE 1 Proportional prevalence of anti-tuberculosis drug-resistance in the International Carbapenems Study Group cohort

\begin{tabular}{|c|c|c|c|c|c|c|}
\hline Cohort & Fluoroquinolones & Clofazimine & Ethambutol & Pyrazinamide & Prothionamide & Kanamycin \\
\hline \multicolumn{7}{|l|}{ International Carbapenems Study Group } \\
\hline \multirow[t]{2}{*}{ New cases $^{\#}$} & $30 / 140$ & & $90 / 140$ & $81 / 130$ & $60 / 137$ & $19 / 84$ \\
\hline & $21.4 \%(14.6-28.2)$ & & $64.3 \%(56.4-72.2)$ & $62.3 \%(53.9-70.6)$ & $43.8 \%(35.5-52.1)$ & $22.6 \%(13.7-31.5)$ \\
\hline \multirow[t]{2}{*}{ Previously-treated tuberculosis cases } & $107 / 195$ & & $140 / 197$ & $113 / 169$ & $113 / 176$ & $80 / 140$ \\
\hline & $54.9 \%(47.9-61.9)$ & & $71.1 \%(64.8-77.4)$ & $66.9 \%(59.8-74.0)$ & $64.2 \%(57.1-71.3)$ & $57.1 \%(48.9-65.3)$ \\
\hline All cases & $\begin{array}{c}137 / 336 \\
40.8 \%(35.6-46.1)\end{array}$ & & $\begin{array}{c}232 / 339 \\
684 \%(635-734)\end{array}$ & $\begin{array}{c}195 / 300 \\
650 \%(596-704)\end{array}$ & $\begin{array}{c}174 / 314 \\
554 \%(499-609)\end{array}$ & $\begin{array}{c}100 / 225 \\
44 . \%(379-509\end{array}$ \\
\hline \multicolumn{7}{|l|}{ Stratification by geographical region } \\
\hline \multicolumn{7}{|l|}{ International Carbapenems Study Group Europe } \\
\hline Previously-treated tuberculosis cases & $61 / 142$ & & $103 / 142$ & $83 / 124$ & $89 / 141$ & 44/87 \\
\hline \multirow{2}{*}{ All cases } & $91 / 283$ & & $\begin{array}{l}12.05 / 284 \\
195 / 2-17.01\end{array}$ & $\begin{array}{c}06.9 \% 138.6-15.21 \\
165 / 255\end{array}$ & & $\begin{array}{l}50.6 \%(40.1-61.1) \\
64 / 172\end{array}$ \\
\hline & $32.2 \%(26.8-37.6)$ & & $68.7 \%(63.3-74.1)$ & $64.7 \%(58.8-70.6)$ & $53.8 \%(48.0-59.7)$ & $37.2 \%[30.0-44.4]$ \\
\hline \multicolumn{7}{|c|}{ International Carbapenems Study Group South America } \\
\hline Previously-treated tuberculosis cases & $\begin{array}{c}46 / 53 \\
86.8 \%(77.7-95.9)\end{array}$ & & $\begin{array}{c}37 / 55 \\
67.3 \%(54.9-79.7)\end{array}$ & $\begin{array}{c}30 / 45 \\
66.7 \%(52.9-80.5)\end{array}$ & $\begin{array}{c}24 / 35 \\
68.6 \%(53.2-84.0)\end{array}$ & $\begin{array}{c}36 / 53 \\
67.9 \%(55.3-80.5)\end{array}$ \\
\hline All cases & $\begin{array}{c}46 / 53 \\
86.8 \%(77.7-95.9)\end{array}$ & & $\begin{array}{c}37 / 55 \\
67.3 \%(54.9-79.7)\end{array}$ & $\begin{array}{c}30 / 45 \\
66.7 \%(52.9-80.5)\end{array}$ & $\begin{array}{c}24 / 35 \\
68.6 \%(53.2-84.0)\end{array}$ & $\begin{array}{c}36 / 53 \\
67.9 \%(55.3-80.5)\end{array}$ \\
\hline \multicolumn{7}{|l|}{ Control } \\
\hline \multicolumn{7}{|l|}{ International Carbapenems Study Group control group } \\
\hline New cases $(108 / 168)$ & $\begin{array}{c}15 / 105 \\
14.3 \%(7.6-21.0)\end{array}$ & & $\begin{array}{c}61 / 105 \\
58.1 \%(48.7-67.5)\end{array}$ & $\begin{array}{c}53 / 96 \\
55.2 \%(45.3-65.1)\end{array}$ & $\begin{array}{c}35 / 103 \\
34.0 \%(24.9-43.1)\end{array}$ & $\begin{array}{c}7 / 56 \\
12.5 \%(3.8-21.2)\end{array}$ \\
\hline Previously-treated tuberculosis cases (59/168) & $\begin{array}{c}12 / 56 \\
21.4 \%(10.7-32.1)\end{array}$ & & $\begin{array}{c}30 / 58 \\
51.7 \%(38.9-64.6)\end{array}$ & $\begin{array}{c}20 / 45 \\
44.4 \%(29.9-58.9)\end{array}$ & $\begin{array}{c}28 / 56 \\
50.0 \%(36.9-63.1)\end{array}$ & $\begin{array}{c}9 / 32 \\
28.1 \%(12.5-43.7)\end{array}$ \\
\hline All cases & 27/161 & & $\begin{array}{c}92 / 164 \\
561 \% 85-6371\end{array}$ & $\begin{array}{c}73 / 141 \\
518 \%(436-60)\end{array}$ & $63 / 159$ & $\begin{array}{c}16 / 88 \\
18 . \%(101-263)\end{array}$ \\
\hline
\end{tabular}

Data are presented as number of resistant cases for a specific drug/total number of cases tested for a specific drug and \% proportional resistance (95\% CI). ${ }^{\#}$ : Out of 145 total new cases, 144 cases were from Europe. Susceptibility to all drugs in the International Carbapenems Study Group was found only in the control group: 14 cases were susceptible to all six drugs out of 168 controls. No data were available for clofazimine. 
toxicities and psychological wellbeing and compliance of the patient, taking into account what WHO recommends to keep the MDR-TB regimen "shorter" $[5,14]$. The importance of cohort discussion and "consilia" for the evaluation of MDR-TB patients could be a useful decisional tool in determining the appropriateness of the shorter MDR-TB regimen and in ensuring its maximum effectiveness [15].

$@$ ERSpublications

Evaluation of drug resistances is needed to identify candidates for the shorter regimen in MDR-TB hot spots http://ow.ly/wZV33022VXt

\begin{abstract}
Giovanni Sotgiu ${ }^{1,22}$, Simon Tiberi ${ }^{2,22}$, Lia D’Ambrosio ${ }^{3,4,22}$, Rosella Centis ${ }^{3,22}$, Jan Willem Alffenaar ${ }^{5,22}$, Jose A. Caminero $^{6,7,22}$, Marcos Abdo Arbex ${ }^{8,9}$, Valentina Alarcon Guizado ${ }^{10}$, Alena Aleksa ${ }^{11}$, Simone Dore ${ }^{1}$, Mina Gaga ${ }^{12}$, Gina Gualano $^{13}$, Heinke Kunst ${ }^{14}$, Marie-Christine Payen ${ }^{15}$, Aurora Jazmín Roby Arias ${ }^{16}$, Alena Skrahina ${ }^{17}$, Ivan Solovic ${ }^{18}$, Giorgia Sulis $\oplus^{19}$, Marina Tadolini ${ }^{20}$, Alimuddin Zumla ${ }^{21,22}$ and Giovanni Battista Migliori $\odot^{3}$ for the International Carbapenem Study Group ${ }^{23}$

${ }^{1}$ Clinical Epidemiology and Medical Statistics Unit, Dept of Biomedical Sciences, University of Sassari - Research, Medical Education and Professional Development Unit, AOU Sassari, Sassari, Italy. ${ }^{2}$ Division of Infection, Barts Health NHS Trust, London, UK. ${ }^{3}$ Fondazione S. Maugeri, IRCCS, Care and Research Institute, Tradate, Italy. ${ }^{4}$ Public Health Consulting Group, Lugano, Switzerland. ${ }^{5}$ Dept of Clinical Pharmacy and Pharmacology, University of Groningen, University Medical Center Groningen, Groningen, The Netherlands. ${ }^{6}$ Pneumology Dept, Hospital General de Gran Canaria "Dr. Negrin", Las Palmas de Gran Canaria, Spain. ${ }^{7}$ MDR-TB Unit, Tuberculosis Division, International Union against Tuberculosis and Lung Disease (The Union), Paris, France. ${ }^{8}$ University Center of Araraquara, Sao Paulo, Brazil. ${ }^{9}$ Hospital Nestor Goulart Reis, Sao Paulo State Secretary of Health, Sao Paulo, Brazil. ${ }^{10}$ National Tuberculosis Control Programme, Ministry of Health, Lima, Peru. ${ }^{11}$ Educational Institution "Grodno State Medical University", Grodno, Belarus. ${ }^{12}$ MDR-TB Unit, Athens Chest Hospital Sotira, National Referral Centre for Mycobacteria, Athens, Greece. ${ }^{13}$ Respiratory Infectious Diseases Unit, National Institute for Infectious Diseases 'L. Spallanzani' IRCCS, Rome, Italy. ${ }^{14}$ Dept of Respiratory Medicine, Queen Mary University, London, UK. ${ }^{15}$ Division of Infectious Diseases, CHU Saint-Pierre, Université Libre de Bruxelles (ULB), Brussels, Belgium. ${ }^{16}$ National Tuberculosis Control Programme, Pulmonology Hospital Alfredo J. Valenzuela, Guayaquil, Ecuador. ${ }^{17}$ Republican Research and Practical Centre for Pulmonology and Tuberculosis, Minsk, Belarus. ${ }^{18}$ National Institute for TB, Lung Diseases and Thoracic Surgery, Vysne Hagy, Catholic University Ruzomberok, Ružomberok, Slovakia. ${ }^{19}$ University Dept of Infectious and Tropical Diseases, WHO Collaborating Centre for TB/HIV Co-infection and for TB Elimination - University of Brescia and Brescia Spedali Civili General Hospital, Brescia, Italy. ${ }^{20}$ Unit of Infectious Diseases, Dept of Medical and Surgical Sciences, Alma Mater Studiorum University of Bologna, Bologna, Italy. ${ }^{21}$ Division of Infection and Immunity, University College London and NIHR Biomedical Research Centre, UCL Hospitals NHS Foundation Trust, London, UK. ${ }^{22}$ These authors contributed equally. ${ }^{23} \mathrm{~A}$ list of members of the International Carbapenem Study Group can be found in the acknowledgements section.
\end{abstract}

Correspondence: Giovanni Battista Migliori, Fondazione S. Maugeri, Care and Research Institute, Via Roncaccio 16, 21049, Tradate, Italy. E-mail: giovannibattista.migliori@fsm.it

Received: June 232016 | Accepted: June 242016 | First published online: Sept 12016

Conflict of interest: None declared.

Acknowledgements: The authors alone are responsible for the views expressed in this article and they do not necessarily represent the decisions and policies of their institutions.

All authors contributed to the conception of the study, data analysis, interpretation of the results, drafting of the manuscript and approval of the final version.

Members of the International Carbapenem Study Group: M. Abdo Arbex (Brazil), O.W. Akkerman (The Netherlands), E. Alarcon Arrascue (Peru), V. Alarcon Guizado (Peru), A. Aleksa (Belarus), J.W. Alffenaar (The Netherlands), J. Artsukevich (Belarus), V. Avchynka (Belarus), J.A. Caminero (Spain), R. Centis (Italy), L. D’Ambrosio (Italy), S. De Lorenzo (Italy), S. Dore (Italy), M. Gaga (Greece), G. Gualano (Italy), A.J. Roby Arias (Ecuador), E.H. Bonini (Brazil), F.A. Chong Marín (Ecuador), G. de Vries (The Netherlands), S. Dore (Italy), H. Kunst (UK), A. Matteelli (Italy), G.B. Migliori (Italy), C. Moschos (Greece), F. Palmieri (Italy), A. Papavasileiou (Greece), M.C. Payen (Belgium), A. Piana (Italy), A. Scardigli (France), A. Skrahina (Belarus), I. Solovic (Slovakia), G. Sotgiu (Italy), A. Spanevello (Italy), G. Sulis (Italy), M. Tadolini (Italy), S. Tiberi (UK), D. Vargas Vasquez (Peru), P. Viggiani (Italy), V. White (UK), A. Zumla (UK).

\title{
References
}

1 World Health Organization. Global Tuberculosis Report 2015. WHO/HTM/TB/2015.22. Geneva, World Health Organization, 2015.

2 Falzon D, Mirzayev F, Wares F, et al. Multidrug-resistant tuberculosis around the world: what progress has been made? Eur Respir J 2015; 45: 150-160.

3 Migliori GB, Sotgiu G, Gandhi NR, et al. Drug resistance beyond extensively drug resistant tuberculosis: individual patient data meta-analysis. Eur Respir J 2013; 42: 169-179.

4 Caminero JA, Scardigli A. Classification of antituberculosis drugs: a new proposal based on the most recent evidence. Eur Respir J 2015; 46: 887-893.

5 WHO treatment guidelines for drug-resistant tuberculosis. 2016 update WHO/HTM/TB/2016.04. Geneva, World Health Organization, 2016.

6 Diel R, Vandeputte J, de Vries G, et al. Costs of tuberculosis disease in the European Union: a systematic analysis and cost calculation. Eur Respir J 2014; 43: 554-565. 
7 Tiberi S, Payen MC, Sotgiu G, et al. Effectiveness and safety of meropenem/clavulanate-containing regimens in the treatment of multidrug and extensively drug-resistant tuberculosis. Eur Respir J 2016; 47: 1235-1243.

8 Tiberi S, Sotgiu G, D’Ambrosio L, et al. Comparison of effectiveness and safety of imipenem/clavulanate-versus meropenem/clavulanate-containing regimens in the treatment of multidrug and extensively drug-resistant tuberculosis. Eur Respir J 2016; 47: 1758-1766.

9 Tiberi S, Sotgiu G, D'Ambrosio L, et al. Effectiveness and safety of imipenem-clavulanate added to an optimized background regimen (OBR) versus OBR control regimens in the treatment of multidrug-resistant and extensively drug-resistant tuberculosis. Clin Infect Dis 2016; 62: 1188-1190.

10 Piubello A, Harouna SH, Souleymane MB, et al. High cure rate with standardised short-course multidrug-resistant tuberculosis treatment in Niger: no relapses. Int J Tuberc Lung Dis 2014; 18: 1188-1194.

11 Van Deun A, Maug AK, Salim MA, et al. Short, highly effective, and inexpensive standardized treatment of multidrug-resistant tuberculosis. Am J Respir Crit Care Med 2010; 182: 684-692.

12 Aung KJ, Van Deun A, Declercq E, et al. Successful '9-month Bangladesh regimen' for multidrug-resistant tuberculosis among over 500 consecutive patients. Int J Tuberc Lung Dis 2014; 18: 1180-1187.

13 Ghimire S, Bolhuis MS, Sturkenboom MG, et al. Incorporating therapeutic drug monitoring into the World Health Organization hierarchy of tuberculosis diagnostics. Eur Respir J 2016; 47: 1867-1869.

14 Tiberi S, D'Ambrosio L, De Lorenzo S, et al. Tuberculosis elimination, patients' lives and rational use of new drugs: revisited. Eur Respir J 2016; 47: 664-667.

15 D'Ambrosio L, Tadolini M, Dupasquier S, et al. ERS/WHO tuberculosis consilium: reporting of the initial 10 cases. Eur Respir J 2014; 43: 286-289.

\title{
Variation in vitamin D deficiency among tuberculosis patients by ethnic group and geographical region of birth: evidence from a diverse south London population
}

\author{
To the Editor:
}

Numerous studies have demonstrated an important association of vitamin D deficiency with tuberculosis (TB) $[1,2]$. Vitamin $\mathrm{D}$ is important for immune function and an appropriate host response to Mycobacterium tuberculosis [3, 4]; 25-hydroxyvitamin D $(25(\mathrm{OH}) \mathrm{D})$ is the primary circulating form of vitamin $\mathrm{D}$ and is used to measure deficiency.

We have previously demonstrated that, although vitamin D deficiency is common in south-east London, patients with TB have significantly lower serum $25(\mathrm{OH}) \mathrm{D}$ than healthy controls matched for age, sex, ethnicity, diet and geographical location [1], with particularly low levels found in TB patients of certain Asian and African ethnicities [2]. Trials of vitamin D supplementation during TB therapy have produced mixed results, particularly with certain vitamin D receptor (VDR) polymorphisms $[5,6]$.

In this study, we aimed to investigate the effect of both ethnicity and region of birth on the $25(\mathrm{OH}) \mathrm{D}$ levels of TB patients living in the UK.

Patients diagnosed with TB between January 2001 and September 2012 at two large south London hospitals were identified retrospectively from the London TB Register and in-house databases. Demographic data, country of birth, year of entry into the UK, TB site (pulmonary or extrapulmonary), treatment dates, 25(OH)D levels and HIV status were extracted.

Patients were included if they had a 25(OH)D level recorded within 6 months before, or 1 month after, starting treatment. Patients with chronic kidney disease on dialysis were excluded.

Country of birth was categorised as Africa (excluding the Horn of Africa); Horn of Africa (Eritrea, Ethiopia, Kenya, Somalia, Sudan); Americas (North and South); Asia (excluding the Indian subcontinent); Indian subcontinent (Bangladesh, India, Pakistan, Sri Lanka); Caribbean; and Europe (including the UK).

Ethnicity was reported by the patient and categories used for analysis were: white, including Irish, European and Latin American; black, including black African, black Other and black Caribbean; Indian Asian for Bangladeshi, Indian and Pakistani; Asian, including Asian, Chinese and Vietnamese; and Other. Patients with missing ethnicity data were recorded as missing. 